\title{
An economic-research-based approach to calculate community health-staffing requirements in Xicheng District, Beijing
}

\author{
Delu Yin ${ }^{1}$, Tao Yin ${ }^{1}$, Huiming Yang ${ }^{1}$, Qianqian Xin ${ }^{1}$, Lihong Wang ${ }^{1}$, Ninyan Li ${ }^{3}$, Xiaoyan Ding ${ }^{2}$
} and Bowen Chen ${ }^{1,2^{*}}$

\begin{abstract}
Background: A shortage of community health professionals has been a crucial issue hindering the development of CHS. Various methods have been established to calculate health workforce requirements. This study aimed to use an economic-research-based approach to calculate the number of community health professionals required to provide community health services in the Xicheng District of Beijing and then assess current staffing levels against this ideal.

Methods: Using questionnaires, we collected relevant data from 14 community health centers in the Xicheng District, including resident population, number of different health services provided, and service volumes. Through 36 interviews with family doctors, nurses, and public health workers, and six focus groups, we were able to calculate the person-time (equivalent value) required for each community health service. Field observations were conducted to verify the duration.

Results: In the 14 community health centers in Xicheng District, 1752 health workers were found in our four categories, serving a population of 1.278 million. Total demand for the community health service outstripped supply for doctors, nurses, and public health workers, but not other professionals. The method suggested that to properly serve the study population an additional 64 family doctors, 40 nurses, and 753 public health workers would be required.

Conclusions: Our calculations indicate that significant numbers of new health professionals are required to deliver community health services. We established time standards in minutes (equivalent value) for each community health service activity, which could be applied elsewhere in China by government planners and civil society advocates.
\end{abstract}

Keywords: Health worker, Community health service, Health professional, Economic-research-based approach, Workload

\section{Background}

China once had an excellent primary healthcare system that was a model for other countries; however, after market reforms in 1978, there was a shift in funding, from community health services (CHS) to specialized hospital-based care, with a mandate for health institutions to generate a large portion of their operating revenue. This led to a proliferation of specialists and the

\footnotetext{
* Correspondence: 1726137684@qq.com

${ }^{1}$ Capital Institute of Pediatrics, 2 YaBao Road, \#328, ChaoYang District, Beijing 100020, China

${ }^{2}$ Community Health Association of China, Chaowai Street. 18 A-2603,

ChaoYang District, Beijing 100020, China

Full list of author information is available at the end of the article
}

excessive use of drugs and high-technology diagnostic tests [1]. Since 1997, China has been committed to reshaping the system of urban CHS. The reforms aimed to position CHS as the foundation and entry point of the health system, supported by new policies and increased government investment. In 1999, 2006, and 2009, the central government issued instructions for the development of CHS in urban areas to support policies and actions [2-5]. Over the last 16 years, great strides have been made. By the end of 2013, the total number of CHS facilities had reached 33965 nationwide, with 8488 community health centers (CHCs) and 25477 community health stations [6], basically achieving the objective of 
"every street has at least one community health center" [7], and the number of community health professionals was 0.42 per 1000 residents, 0.08 more than in 2005 [6].

Yet, health human resources and population need has slowed the growth of CHCs. A shortage of community health professionals has been a crucial issue hindering the development of CHS $[8,9]$. In 2006, the central government issued a standard for configuring health staff in urban communities to respond to the dilemma, which, to some extent, promoted the creation of community health professionals [10]. According to the standard, each $\mathrm{CHC}$ will configure two to three family doctors, one public health worker for each 10000 head of population, and the ratio of doctors and nurses should reach $1: 1$, while the proportion of the other professionals accounts for less than $5 \%$ of the total [10]. In recent years, CHS have been further recognized and used by local residents, and the workload of community health professionals has become much higher than that of specialists in hospitals. In 2013, family doctors routinely saw 15.7 outpatients each day, while hospital doctors saw an average of 7.3 [6]. Moreover, in 2009, the Chinese government issued guidance on a National Essential Public Health Services Package (NEPHSP), aiming to provide similar public health services regardless of geographical area, and to expand the coverage of essential public health programs to all residents of China [11]. The NEPHSP greatly increased the workload of community health professionals. With the increased requirements on community public health services and the loss of community health professionals as a result of ineffective incentive mechanisms [12], demand for CHS could no longer be met, and the situation has steadily worsened. This has already affected the performance of the CHS system and hindered its crucial role as the gatekeeper of the health system [13]. An evaluation on programs regarding the community-based management of hypertension and type 2 diabetes mellitus patients in eight provinces showed the standardized management rates of patients with hypertension/type 2 diabetes mellitus patients were only 61.1 and $59.0 \%$ in 2014 [14]. Community health professionals need to work overtime for 1.5-2 h. The professionals' satisfaction with income and workload were lowest, respectively 45.23 and $45.65 \%$ [15]. While there has been guidance provided about the ideal configuration of community health staff in urban communities, a decade ago, there has been little done more recently to understand the health human resource requirements for $\mathrm{CHCs}$ given the NEPHSP.

\section{Measuring workforce requirements}

Various methods have been established to calculate health workforce requirements. Many use practitionerto-population ratios, or historical patterns [16, 17], although there are other methods including case-load profiling, acuity measures, queuing theory, and production function [18-21]. In 1998, the World Health Organization (WHO) developed the Workload Indicators of Staffing Need (WISN) to calculate optimal allocations and use of staff [22-24]. Compared with the other methods, its results are relatively reasonable and accurate, which is helpful for healthcare workforce planning [23]. The method has previously been used in Tanzania, Namibia, and elsewhere. The problem with the WISN approach is the static and narrowly focused model underlying the WHO approach $[20,25]$.

\section{Purpose of this research}

The purpose of this project was to use an economicresearch-based approach to calculate the demand and supply of community health professionals in Xicheng District and to measure the staffing gap between the ideal number and current staffing levels. This provided a reference point and effective basis for assessing community health-staffing issues and formulating relevant policies in other areas in China and elsewhere.

\section{Methods}

\section{Study setting}

The study focused on the Xicheng District in Beijing City, the capital of China. The district is located in downtown Beijing and has 15 streets of offices and 255 community neighborhood committees. In 2013, the population in Xicheng District was 16200000 , with a GDP of about USD 40.3 billion. In total, there are 620 health facilities in the district, including 15 tertiary hospitals, 14 secondary hospitals, and 78 community health stations affiliated to $15 \mathrm{CHCs}$. Currently, there are 2035 personnel working in community health facilities, of which $82.10 \%$ are community health professionals.

\section{Data collection}

We focused our study on all types of services provided to the residents and restricted community health professionals to family doctors, nurses, public health workers, and other professionals including pharmacists and laboratory technicians. The study was further limited to 14 government-owned $\mathrm{CHCs}$ and their affiliated community health stations. The remaining $\mathrm{CHC}$ was hospital-owned and refused to provide data for the study. To ensure the on-site surveys and data collection could be done smoothly, a six-member research team was set up, including the health administration officials of Xicheng District, experts in healthcare human resources, agency officials, doctors, nurses, and public health physicians, supported by the local health administration department. Oral consent was provided by the 
health professionals and agency heads before their participation in any discussions or interviews.

Data for the calculation were collected as part of a larger study from 14 CHCs in Xicheng District in 2013. A standardized form was used to collect basic information, including the resident population, current number of community health professionals, and volume of each type of CHS. CHC managers were trained on how to collect the necessary data. Data were collected from the CHS information system over a 2-week period. Two separate researchers conducted site visits to oversee the data collection.

\section{Demand calculations}

A report on the performance evaluation of CHS nationwide conducted in 2011-2013 showed that the quality of some CHS was lower than required in the guidelines because of a shortage of community health professionals [26]. We calculated the total demand for CHS that would be generated if all the services were delivered at the level of quality set out in the medical, nursing, and public health guidelines. We then estimated the total workload indicator (person-time) required to provide all the CHS for the study population for the period from January 1 to December 31, 2013. Demand was also measured based on the actual volume of CHS used by the residents in 2013 in the sample areas.

\section{What components are included in community health services?}

China's community health facilities were designed to deliver comprehensive primary healthcare services, from basic medication to rehabilitation, and public health services including the NEPHSP, which covers health education, children's health services and immunization, maternal health services, older people's health management, and services for patients with chronic diseases [27, 28]. According to research conducted in 2014, public health work accounts for about $45 \%$ of the total workload in urban community health facilities nationwide [29]. To calculate demand, we catalogued all these types of CHS and classified them into five categories by staffing requirements: essential medical services, nursing, pharmacy, auxiliary examinations, and public health services.

\section{How much time does it take to deliver each service unit?} We estimated the workload indicator for each service unit based on two steps: (1) determining the standard service protocols of all types of CHS and (2) defining the workload indicator for a set of standard activities for each service, and their equivalent value (EV) compared with a standard clinic visit. This method has been used to estimate the cost of the National Essential Public Health Services Package in Beijing, China [30].
Step 1: determining the standard service protocols All types of CHS provided by the sampled CHCs were investigated. In total, 134 types of $\mathrm{CHS}$ were deemed necessary for inclusion in the standard service protocols, including medical (35), nursing (15), paramedical (5), public health services (65), and auxiliary examinations (14). The main types are listed in Table 1. Only 10 of the 134 were included in the 2011 NEPHSP guidelines [31]. The remaining 124 types of CHS were defined in line with the 2007 Beijing technical specification for CHS [32].

\section{Step 2: calculating the workload indicator and the EV of each CHS}

To create the workload indicator (person-time) for each CHS, a multi-stage iterative feedback and revision process was conducted. A series of four meetings were held with 42 invited participants with particular knowledge and expertise about CHS. Attendees included 14 CHS managers, 14 family physicians, 6 nurses, and 8 public health workers. During the meetings, participants discussed the staffing and time requirements for each $\mathrm{CHS}$, the workload assigned to each in the standard service protocols, and possible modifications to this.

To test these workload indicators, $6 \mathrm{CHCs}$ were randomly selected from the sample of 14 to participate in direct observations. Five research assistants were trained to observe the services and record the length of time for each CHS and the number and staff group of health workers involved. Direct observation took place over a period of three continuous days in each $\mathrm{CHC}$. Face-toface interviews were conducted to determine the usual time and the required number of health workers for each service, to check against the direct observations.

The workload indicators were modified based on the direct observation and interviews. Group interviews with 20 staff at the eight centers were conducted to test the workload indicators. Six family physicians, four nurses, seven public health workers, and three other health professionals participated in the interviews.

To ensure that the different types of CHS can be directly compared, a "standard clinic visit" was introduced as a benchmark to gauge the necessary staff and time required (workload indicator) for the other services. A standard clinic visit was defined as a family physician consulting with one patient for 15 min [33]. The workload indicator of a standard clinic visit was defined as one EV. EVs for all other CHS were then calculated by comparing their workload indicator from step 2.1 with the standard clinic visit. For example, a home visit may include the time taken to travel to and from the patient's home and to administer medication. The workload indicator of one home visit was $60 \mathrm{~min}$ in both urban and 
Table 1 Workload and EV of the main CHS compared with a standard clinic visit

\begin{tabular}{|c|c|c|c|}
\hline Categories & Types & Workload (minutes) & Mean EV \\
\hline \multirow[t]{5}{*}{ Essential medical services } & Single clinic visit & 15.00 & 1.00 \\
\hline & Emergency (per visit) & 37.50 & 2.50 \\
\hline & Single home visit & 60.00 & 4.00 \\
\hline & Inpatient bed day & 90.00 & 6.00 \\
\hline & Rehabilitation clinic (per outpatient visit) & 40.00 & 2.67 \\
\hline \multirow[t]{5}{*}{ Nursing services } & Intravenous injection & 5.00 & 0.33 \\
\hline & Intravenous infusion & 12.00 & 0.80 \\
\hline & Intravenous injection, venous blood & 7.00 & 0.47 \\
\hline & Catheterization & 20.00 & 1.33 \\
\hline & Providing prescription (Western medicine) & 3.00 & 0.20 \\
\hline \multirow[t]{2}{*}{ Pharmacy service } & Dispensing a prescription (per prescription) & 12.50 & 0.83 \\
\hline & Advanced pharmacy work, including detailed dosage calculations (per prescription) & 75.00 & 5.00 \\
\hline \multirow[t]{5}{*}{ Auxiliary examination service } & Rapid blood sugar test & 3.00 & 0.20 \\
\hline & Blood, urine, feces test (per test) & 13.00 & 0.87 \\
\hline & Biochemical test (per test) & 30.00 & 2.00 \\
\hline & Electrocardiogram (per test) & 5.00 & 0.33 \\
\hline & B-mode ultrasonography (per test) & 15.00 & 1.00 \\
\hline \multirow[t]{9}{*}{ Public health service } & Health record management service (per person year) & 50.00 & 3.33 \\
\hline & Health education service (per month) & 1400.00 & 93.33 \\
\hline & Health services for children aged 0 to 36 months (per person year) & 106.67 & 7.11 \\
\hline & Maternal health services (per person year) & 296.00 & 19.73 \\
\hline & Older people's health services (per person year) & 70.00 & 4.67 \\
\hline & Immunizations (per visit) & 26.55 & 1.77 \\
\hline & Patients with hypertension (per person year) & 310.00 & 20.67 \\
\hline & Patients with type II diabetes (per person year) & 310.00 & 20.67 \\
\hline & Patients with severe mental illness (per person year) & 510.00 & 34.00 \\
\hline
\end{tabular}

suburban areas, so its EV was $4(60 \div 15)$. The EV of each CHS is shown in Table 1.

\section{What is the total workload required to serve the study population, by staff group?}

The volume of each service in 2013 was multiplied by the EV for each, and these figures were summed to find the total EVs for all 134 types of CHS across the sampled $\mathrm{CHCs}$. This gave a figure for the total workload required to serve the study population. Nurses generally had broad responsibilities extending beyond traditional nursing roles, including involvement in the provision of basic medication and public health services. The group interview participants estimated that nurses spent $30 \%$ of their time on public health services.

\section{Supply calculations}

We calculated the available supply of community health professionals in the $14 \mathrm{CHCs}$. We multiplied days worked by working hours per day and subtracted holidays, weekends, meal times, and rest time for each working day. Based on the interviews with community health professionals, each health professional generally works for 250 days in a year, for six effective working hours per day. The EVs per year of a full-time professional are therefore $6000 \mathrm{EVs}(=(250$ days $\times 6 \mathrm{~h} \times$ $60 \mathrm{~min}) \div 15 \mathrm{~min}$, because $15 \mathrm{~min}$ is the workload for one EV). We then used the following formula to calculate the supply:

$$
\begin{aligned}
& \text { Supply } \\
& =\sum \text { Number of community health professionals in the } 14 \mathrm{CHCs} \\
& \times 6000 \text { EVs. }
\end{aligned}
$$

\section{Gap and surplus calculations}

We subtracted total demand EVs from total supply EVs to derive the gap or surplus EVs available for community health professionals in the 14 centers. We then divided these gap or surplus EVs by the number of EVs per year 
that a full-time professional would expect to work, to determine the equivalent number of full-time community health professionals required to fill the gap or in excess. We then calculated the ratio of total supply EVs to demand EVs to estimate the magnitude of the gap or surplus. A ratio of 1 implies perfect balance, below 1 indicates a gap and above 1 indicates a surplus.

\section{Results}

\section{Staffing sample CHCs}

Each sample CHC had an average of 125 employees on the payroll (1752 employees in 14 centers), serving an average community of 91400 people (a total of 1.278 million people). The majority of community health professionals were doctors (49.6 people per center), followed by nursing staff (37.1), then public health workers (19.7). There were 18.6 other community health professionals per center, including pharmaceutical, imaging, and inspection personnel, but not logistics or administrative staff.

\section{Demand calculations}

For the $14 \mathrm{CHCs}$ in Xicheng District, the total demand in 2013 was about 17.18 million EVs. Public health services made up $51.3 \%$ of the total demand, which more than basic medical services (see Table 2). The average EVs for each center were 1226857.

\section{Supply calculations}

The current number of healthcare workers in post was 1752 , while the annual supply of a full-time professional is 6000 EVs. The supply available in the 14 CHCs of Xicheng District is therefore approximately 10512000 EVs (Table 3).

\section{Calculation of "gap/surplus"}

Table 3 illustrates the demand and supply calculations for each category of community health professionals and

Table 2 CHS demand by category of service across 14 sampled $\mathrm{CHCS}$

\begin{tabular}{|c|c|c|}
\hline Categories & Demand (EVs) & Percentage \\
\hline Basic medical services & 4556299.2 & 26.5 \\
\hline Nursing services & 710181.84 & 4.1 \\
\hline Pharmacy services & 1271402.8 & 7.1 \\
\hline Auxiliary examinations & 257315.52 & 1.5 \\
\hline Public health services & 8819343.5 & 51.3 \\
\hline Other services $^{a}$ & 1561454.3 & 10.0 \\
\hline Total & 17175997 & 100 \\
\hline
\end{tabular}

${ }^{a}$ These services cannot be included in any category above. Administrative and other duties (report writing, continued professional education sessions and meetings, etc.) allocated to each category of community health professionals were included as well. Group interviews suggested their workload could account for $10 \%$ of the total the gap or surplus for each. The ratio of supply to demand in the final column shows the magnitude of the shortage or surplus. For example, the total demand for basic medical services was 4556299.23 EVs. However, 695 doctors can only supply $4170000 \mathrm{EVs}$, so the gap is 386299 EVs. To staff the gap, an extra 64 doctors is needed, giving a ratio of 0.92 . As Table 3 shows, the total demand for CHS was greater than could be supplied for doctors, nurses, and public health workers. Providing the study population with the required standard of health services would need an additional 64 GPs, 40 nurses, and 753 public health workers. The category of other professionals, such as laboratorians and pharmacists, appears to be slightly over-staffed, with a ratio of supply to demand of about 1.03 .

\section{Discussion}

We used an economic-research-based approach to explore the capacity of community health professionals to meet demand for services in $14 \mathrm{CHCs}$ in Xicheng District, Beijing. We created lists of CHS and estimated the person-time (equivalent value) for each service unit, based on a multi-stage iterative feedback and revision process. The total demand for CHS was calculated from the actual volume of CHS used by the residents of the sample areas in 2013. The total supply for CHS was calculated using staffing data supplied by the sample CHCs.

The study shows that the total supply and demand ratio for community health professionals in Xicheng District was 0.67 , which suggests a significant unmet demand for services or overworking of human resources in CHS. This may be attributed to the greatly improved use of essential medical services and China's national equalization of basic public health services in 2009. If the government of Xicheng intends to meet resident demand for CHS, and enable it to act as the gatekeeper of the health system, it will need to deliver significant improvements in CHSstaffing levels. Family doctors and nurses are in slight shortage, with a ratio of 92 and 93\%, respectively. A 10\% work pressure is always allowed, and therefore, it means the ratio for family doctor and nurses is fine as long as it is evenly distributed. Redistribution of current family doctor and nurses is recommended if the ratio varies across facilities. The current numbers of public health workers are a particular issue, being only $27 \%$ of the staff required to meet demand. In practice, because of the shortage of public health workers, $30 \%$ of nurses' working time was estimated by the professionals involved to be allocated to the provision of public health services. Generally, one doctor potentially will generate more nursing work than one nurse can handle. It is particularly more significant that the doctor/nurse ratio be nearly 1:1 given that the nurses are providing public health services in addition to nursing care. This has led to concerns about the quality and 
Table 3 Demand, supply, gap, and surplus in CHS provision in Xicheng District, Beijing

\begin{tabular}{|c|c|c|c|c|c|c|}
\hline & $\begin{array}{l}\text { No. of community health } \\
\text { professionals on the job (a) }\end{array}$ & $\begin{array}{l}\text { Total demand in EV for } \\
\text { CHS (b) }\end{array}$ & $\begin{array}{l}\text { Total supply } \\
\text { in EV for CHS } \\
(c=a \times 6000)\end{array}$ & $\begin{array}{l}\text { Gap/surplus in EV ( } d \\
=c-b)\end{array}$ & $\begin{array}{l}\text { No. of additional professionals } \\
\text { required (6000 EVs per year } \\
\text { per FTE employee) (d/6000) }\end{array}$ & $\begin{array}{l}\text { Ratio } \\
(e=c / b)\end{array}$ \\
\hline $\begin{array}{l}\text { Basic medical } \\
\text { services }\end{array}$ & 695 & 4556299.23 & 4170000 & -386299 & 64 & 0.92 \\
\hline Nursing services & 519 & 3355984.89 & 3114000 & -241985 & 40 & 0.93 \\
\hline $\begin{array}{l}\text { Public health } \\
\text { services }\end{array}$ & 276 & 6173540.45 & 1656000 & -4517540 & 753 & 0.27 \\
\hline Other services ${ }^{a}$ & 262 & 1528718.36 & 1572000 & 43282 & 7 (surplus) & 1.03 \\
\hline Total & 1752 & 15614543 & 10512000 & -5102543 & 850 & 0.67 \\
\hline
\end{tabular}

aOthers include pharmacy services, auxiliary examination services, and services that cannot be included

deliverability of the NEPHSP and the nursing care as well. The calculation was based on the existing volume of service demand, quality service reaching the standard requirements, and no working overtime of professionals. Thus, the approach based on using existing levels of demand found a provider-shortage demand. The extra providers needed to improve the current poor quality and solve the problem of professionals working overtime which constituted the shortage of community health professionals.

Although the results of this study were obtained based on the actual situation in Xicheng District, they are also likely to reflect wider supply and demand imbalances among China's urban community health professionals. Governments and relevant departments at all levels should therefore pay close attention to this issue. The nationwide implementation of the NEPHSP was designed to address increasing public health problems and improve the equity of China's health service [11]. However, it did greatly increase the workload of primary healthcare facilities [34]. Effort is now needed to increase the numbers of public health workers in urban community health facilities and village hospitals in rural areas. It seems likely that use of CHS will increase over the next few years, with a series of supportive policies issued $[6,35,36]$, and that the NEPHSP will expand to include more services and cover larger populations [29]. Additionally, the calculation was built on the assumption that the present way of delivering care was the most efficient way of delivering care and the existing levels of provider productivity are acceptable. With the improvement of the service delivery model and service productivity, the demand of community health professionals will change. This risks the development of further imbalances in supply and demand. A dynamic community health professional adjustment mechanism should therefore be established. Using this, the demand and supply of community health professionals, and the size of any gap or surplus, could be tracked in real time to enable timely action to address the problem.

This study did have some limitations. First, the approach primarily measures effort, but neglects issues of efficiency and effectiveness. More attention will be directed at the efficiency and effectiveness of various workers and combinations of workers providing the same service. Second, this study was based only in one location, a central urban region with a high population density. The results might be different in regions with different characteristics, such as low population density, or rural areas, especially those with mountains or forests. Future studies should examine different types of area. To our knowledge, this is the first time that the approach has been used to study the healthcare workforce in Chinese primary healthcare settings, and we suggest that the time standard and EV for each type of CHS proposed in this study could be applied to other similar districts in China and elsewhere.

\section{Conclusions}

This study used an economic-research-based approach to calculate the number of community health professionals required to provide CHS in the Xicheng District of Beijing and then assessed the current staffing levels against this ideal. Our calculations indicated that significant numbers of new health professionals are required to deliver CHS. The current numbers of public health workers are a particular issue, being only $27 \%$ of the staff required to meet demand. A dynamic community health professional adjustment mechanism should be established with the increasing use of $\mathrm{CHS}$ and the improvement of service delivery model and service productivity.

\footnotetext{
Acknowledgements

We would like to thank the Center for Community Health Service Management in Xicheng District, Beijing, for their generous support. We are also grateful to Professor Sabrina T. Wong (University of British Columbia, School of Nursing and Centre for Health Services Policy Research) and Dr. Chen Wen-Qiang (LKS Faculty of Medicine, The University of Hong Kong) for providing valuable suggestions about both language and content during the preparation and revision of this manuscript.
}

\section{Authors' contributions}

$Y D, Y T, Y H, X Q, L N, W L, D X$, and $C B$ were responsible for the conception, design, and acquisition of the data. YD was responsible for the analysis and interpretation of the data and drafting the initial manuscript. YT, YH, XQ, LN, $W L$, and DX revised the manuscript critically for important intellectual content. CB was responsible for reviewing all drafts of the manuscript and 
giving final approval of the version to be published. All authors read and approved the final manuscript.

\section{Competing interests}

The authors declare that they have no competing interests. Natural Science Foundation of China (71303164)

\section{Author details}

'Capital Institute of Pediatrics, 2 YaBao Road, \#328, ChaoYang District, Beijing 100020, China. ${ }^{2}$ Community Health Association of China, Chaowai Street. 18 A-2603, ChaoYang District, Beijing 100020, China. ${ }^{3}$ Center for Community Health Service Management in Xicheng District of Beijing, 8 SanYi DongLi, Xicheng District, Beijing 100055, China.

Received: 7 March 2016 Accepted: 8 September 2016

Published online: 07 December 2016

\section{References}

1. Hsiao WC, Liu Y. Economic reform and health: lessons from China. N Engl J Med. 2006:335:430-2.

2. BSC. Notice about several opinions on the development of city community health services. Beijing: Beijing State Council; 1999.

3. BSC. Guiding opinion on developing urban community health services. Beijing: Beijing State Council; 2006.

4. CCPCSC. Guiding opinion on reform of health system in China. Beijing: The Central People's Government of the People's Republic of China; 2009.

5. Chen BD, Guo A, Yin D. Promoting universal primary health services in China through general practice reforms. The contribution of family medicine to improving health systems: a guidebook from the World Organization of Family Doctors. Geneva: World Organization of Family Doctors (WONCA); 2013.

6. $\mathrm{MOH}$. Chinese health statistical yearbook 2013. Beijing: Ministry of Health of the People's Republic of China; 2014.

7. SCOPSR. Notice on issuing guiding opinions on setting and staffing standards of urban community health care service institutions. Beijing: State Commission Office of Public Sector Reform; 2006.

8. Huang CC, Xiaoming P. State of development of community health services and thinking about existing problems. Chin Health Serv. 2004;20:290-1.

9. Liu L. Situation and challenges of community health service in China. Chin Nurs Manag. 2011;11:6-8.

10. $\mathrm{MOH}$. Guidance on allocation standards for community health services in urban China. Beijing: Ministry of Health of the People's Republic of China; 2006.

11. MOF. Opinion on promoting gradual equalization of the essential public health services. Beijing: Ministry of Finance of the People's Republic of China; 2009

12. Zhang Xintong W. Loss of community health workers and countermeasures based on the integrated incentive model. Chin Gen Pract. 2014;17:4052-5.

13. Hu Y. Difficulties and countermeasures of community health institutions in the new period. China Med Herald. 2014;16:154-8.

14. Li Y, Ren D, Ding P, Zhang Q, Zhang J, Shi W, Wu J, et al. Evaluation on programs regarding the community-based management of hypertension and type 2 diabetes mellitus patients in eight provinces, China. Chin J Epidemiol. 2014;35:35-9.

15. CHAC. Evaluation report on the national essential public health services package. Beijing: Community Health Association of China; 2014.

16. Markham B, Birch S. Back to the future: a framework for estimating healthcare human resource requirements. Can J Nurs Adm. 1997:10(1):7-23.

17. Daviaud E, Chopra M. How much is not enough? Human resources requirements for primary health care: a case study from South Africa. Bull World Health Organ. 2008:86:46-51.

18. Hurst K. Primary and community care workforce planning and development. J Adv Nurs. 2006;55(6):757-69.

19. Hurst K, Smith A, Casey A, Fenton K, Scholefield H, Smith S. Calculating staffing requirements. Nurs Manag (Harrow). 2008;15(4):26-34.

20. Musau P, Nyongesa P, Shikhule A, Birech E, Kirui D, Njenga D, et al. Workload indicators of staffing need method in determining optimal staffing levels at Moi Teaching and Referral Hospital. East Afr Med J. 2008:85(5):232-9.

21. Schoo AM, Boyce RA, Ridoutt L, Santos T. Workload capacity measures for estimating allied health staffing requirements. Aust Health Rev. 2008;32(3):548-58.
22. WHO. Workload indicators of staffing need: a manual for implementation. Geneva: World Health Organization; 1998.

23. Hagopian A, Mohanty MK, Das A, House PJ. Applying WHO's 'workforce indicators of staffing need' (WISN) method to calculate the health worker requirements for India's maternal and child health service guarantees in Orissa State. Health Policy Plan. 2012;27:11-8.

24. Guilbert JJ. The World Health Report 2006: working together for health. Educ Health (Abingdon). 2006;19(3):385-7.

25. McQuide PA, Kolehmainen-Aitken RL, Forster N. Applying the workload indicators of staffing need (WISN) method in Namibia: challenges and implications for human resources for health policy. Hum Resour Health. 2013;11(1):64

26. CHAC. Report on the performance of community health services in urban China. Beijing: Community Health Association of China; 2013.

27. Bhattacharyya O, Delu Y, Wong ST, Chen B. Evolution of primary care in China 1997-2009. Health Policy. 2011;100(2):174-80.

28. Wong ST, Yin D, Bhattacharyya O, Wang B, Liu L, Chen B. Developing a performance measurement framework and indicators for community health service facilities in urban China. BMC Fam Pract. 2010;11(1):91.

29. CHAC. Estimating the cost of the National Essential Public Health Services Package. Beijing: Community Health Association of China; 2014.

30. Yin D, Wong ST, Chen W, Xin Q, Wang L, et al. A model to estimate the cost of the National Essential Public Health Services Package in Beijing, China. BMC Health Serv Res. 2015:15:222.

31. MOH. Guideline of National Essential Public Health Services (2011 version) Beijing: Ministry of Health; 2011.

32. BHB. Beijing technical specification of primary health services. Beijing: Beijing Health Bureau; 2007.

33. $\mathrm{MOH}$. General practitioner standardization training outline (on trial). Beijing: Ministry of Health; 2009

34. Wang F, Ly B, Ding X, Dai T. The national essential public health services project in China: progress and equity. Chin J Health Policy. 2013;6:9-14.

35. MOH: Ministry of Health. Chinese health statistical yearbook 2011. Beijing: Ministry of Health; 2012

36. MOH: Ministry of Health. Chinese health statistical yearbook 2012. Beijing: Ministry of Health; 2013

\section{Submit your next manuscript to BioMed Central and we will help you at every step:}

- We accept pre-submission inquiries

- Our selector tool helps you to find the most relevant journal

- We provide round the clock customer support

- Convenient online submission

- Thorough peer review

- Inclusion in PubMed and all major indexing services

- Maximum visibility for your research

Submit your manuscript at www.biomedcentral.com/submit 